\title{
A prospective audit into the proportion of hospitalised oncology and haematology patients at risk of refeeding syndrome
}

\author{
L. Graham, S. Gupta, H. Sadia, N. El-Mahdawi, V. Agarwal and M. Butt \\ The Queens Centre of Oncology and Haematology, Castle Hill Hospital, Castle road, Cottingham, East Yorkshire \\ HU16 5JQ, UK
}

Refeeding syndrome (RFS) has been recognised as a serious biochemical condition resulting from sudden refeeding of malnourished patients. It is characterised by intracellular loss of electrolytes and profound hypophosphataemia, though there is no concrete definition $^{(1,2)}$. In addition to hypophosphataemia, hypokalaemia and hypomagnesaemia are also common ${ }^{(3)}$. Patients with various types of malignancies are at-risk of RFS due to treatment side effects and disease progression; however, very little exists in the oncologic literature about this disorder ${ }^{(3)}$.The aim of this audit was to identify the proportion of hospitalised oncology and haematology patients who are at risk from this potentially fatal condition.

In this audit, RFS was defined as "A collection of electrolyte disturbances, including one or more of the following: hypophosphataemia $(<0.7 \mathrm{mmol} / \mathrm{l})$, hypokalaemia $(<3.5 \mathrm{mmol} / \mathrm{l})$, hypomagnesia $(<0.5 \mathrm{mmol} / \mathrm{l})$ occurring $2-4$ days post dietetic intervention i.e. oral nutrition support, enteral or parenteral nutrition". A prospective observational audit was undertaken over 3 months (January-March 2009); approximately 1400 new patients were admitted to the oncology centre during this time. All newly admitted patients were screened for risk of malnutrition by qualified nursing staff, using a Trust validated screening tool ${ }^{(4)}$. Of the 1400 new inpatients, $154(11 \%)$ had a nutritional risk score $>10$ (high risk) and were referred to the dietetic department, $95(62 \%)$ were included in the audit. Sixteen (10\%) patients were excluded due to death (unrelated to RFS) or entering the terminal stages of illness. Forty three (28\%) patients were omitted from the audit due to time constraints. Registered Dietitians identified patients who were at a high risk of RFS, medical staff were notified and Vitamin $\mathrm{B}_{1}$ supplementation requested ${ }^{(5)}$.

The mean age of the patients $(n=95)$ was 65 years (SD 12), mean weight $65 \mathrm{~kg}$ (SD 16) and mean BMI 21 (SD 8 ). Thirty two percent $(n=31)$ of the patients were identified as being at high risk of developing RFS. Based on the audit definition, 45\% ( $n=14)$ of the patients identified as high-risk by the dietetic team were found to have developed RFS, four patients had haematological malignancies, 3 head and neck cancer, 3 rectal cancer, 3 lung cancer and 1 prostate cancer. Despite NICE guidelines there was a reluctance to prescribe Vitamin $B_{1}$ supplementation in $35 \%$ of cases.

Forty five percent of oncology and haematology patients developed RFS after being identified as high risk. RFS is an under recognised serious biochemical condition, which can complicate the treatment of poor nutritional status. Early dietetic involvement to aid recognition of at risk patients, combined with medical staff vigilance is imperative in the management of this under-recognised condition.

1. Crook M \& Swaminathan R (1996) Ann Clin Biochem 33, 376-396.

2. Hearing SD (2004) BMJ 328, 908-909 (doi: 10.1136/bmj.328.7445.908).

3. Marinella MA (2008) Int J Clin Pract 62, 460-465.

4. McDougall T, Knight S, Kirkwood B et al. (2008) J Clin Nurs 17, 2791-2792.

5. National Institute for Health and Clinical Excellence (NICE). (2006) Nutrition Support in Adults: Oral Nutrition Support, Enteral Tube Feeding and Parenteral Nutrition. Clinical Guideline 32. 